\title{
Los 'j-blogs': la columna periodística se transforma en los diarios digitales y quiere disfrazarse de bitácora
}

\author{
Félix PÉREZ BAHÓN \\ Universidad Carlos III de Madrid \\ bahon30@gmail.com
}

Recibido: 09/10/2012

Aceptado: 23/01/2013

\section{Resumen}

Una de las primeras consecuencias del proceso de integración redaccional a que se han visto sometidos los principales diarios españoles ha sido la eclosión en sus cabeceras digitales de "blogs periodísticos" también conocidos como "j-blogs" (journalistic blogs)- firmados por profesionales de los propios medios. Este trabajo de carácter exploratorio y explicativo pone de manifiesto que hay un denominador común en todos ellos: repiten características y funciones de las tradicionales columnas de opinión y, sin embargo, presentan elementos diferenciales que los alejan del patrón estilístico y discursivo de la blogosfera, al menos tal y como la han entendido en los últimos años los investigadores de la comunicación. Palabras clave: blogs periodísticos, géneros argumentativos, internet, ciberperiodismo

\section{The "j-blogs": the Journalistic Column Is Transformed Into Digital Newspapers But Wants to Masquerade as a Blog}

\begin{abstract}
One of the first consequences of the editorial integration process in the main Spanish newspapers has been the explosion of the " $\mathrm{j}-\mathrm{blogs}$ " by media professionals in their digital headers. There is a common denominator for all of them, repeating features, functionality of traditional opinion. There is a distinct difference in the style and pattern that moves away from the "blog" format.

Keywords: journalistic blogs, argumentative genres, Internet, online journalism

Referencia normalizada

PÉREZ BAHÓN, Félix (2013): “Los 'j-blogs': la columna periodística se transforma en los diarios digitales y quiere disfrazarse de bitácora". Estudios sobre el mensaje periodístico. Vol. 19. Núm. especial abril, págs.: 915-923. Madrid, Servicio de Publicaciones de la Universidad Complutense.
\end{abstract}

Sumario: 1. Introducción. 2. Marco referencial y formulación del problema. 3. Objetivo, hipótesis y metodología. 4. Desarrollo de la investigación. 5. Conclusiones. 6.. Referencias bibliográficas.

\section{Introducción}

Internet impone nuevas reglas para seleccionar, producir y difundir la información. Los cambios son tan acelerados que académicos y analistas se ven empujados a volver una y otra vez a la palestra para debatir sobre nuevos paradigmas comunicativos y transformación del quehacer profesional. Una de las cuestiones sometidas al constante proceso de revisión es la relación entre blogs y medios de comunicación. La novedad ahora la aportan por un lado los protagonistas, periodistas tradicionales con gran experiencia y prestigio aunque novatos en la plataforma digital, y por otro, el escenario: blogs asociados a la página web de diarios de referencia en papel. 


\section{Marco referencial y formulación del problema}

Probablemente el documento más importante sobre blogs y periodismo lo ofreció la Universidad de Harvard en 2003 con un Nieman Reports que recoge una veintena de ensayos de Blood, Lasica, Gillmor, Allbritton, Grabowicz, etc. En ese momento en los medios españoles apenas se planteaba "el debate banal" acerca de si los blogs eran periodismo o no (Noguera, 2008). El miedo a que páginas personales y medios sociales pudieran sustituir a los periodistas profesionales y que acabaran definitivamente con una industria editorial en crisis apoyó la resistencia de los profesionales frente a la red.

Hasta 2005, artículos y libros se centran en definiciones preliminares e intentos de acotar el fenómeno blog como un nuevo medio llegado de la mano de la plataforma digital (Fernández Parrat, 2001; Blood, 2002: 19; Gillmor, 2004; Orihuela, 2005: 7082). Los esfuerzos se centran en identificar el objeto sobre el que se debate (Andrews, 2003) así como en observar la función social o profesional que pueden desarrollar (Edo, 2002; Blood, 2003; Lasica 2003; García y Capón, 2004.

A partir de 2005, ya superados primeros prejuicios y con las webs de los diarios españoles abiertas a las bitácoras, se produce una avalancha de bibliografía. Trabajos de campo elaboran una taxonomía de la blogosfera hispana, describen sus herramientas y proponen estilos y normas (Estalella, 2005; López García, 2005: 133-147; Orihuela, 2006; Flores y Aguado, 2006; Marcelo y Martín, 2007). Incluso hay autores que consideran que estamos hablando de un nuevo género periodístico (Larrondo-Ureta 2005; Salaverría, 2005: 155-157; Morán, 2005).

En un tercer periodo (desde 2005), los investigadores han demostrado un especial empeño en analizar específicamente las características y funciones de los llamados "blogs periodísticos", conocidos como "j-blogs" (journalistic blogs) (Rengel, 2005; Noguera, 2008; Salaverría, 2008; Palomo y Meso, 2009; Sanchez Vigil et al, 2010). Para Ramón Salaverría, en concreto, estas investigaciones organizan estas páginas en tres grupos:

a) Bitácoras elaboradas por periodistas profesionales en plantilla y alojadas en la página web del medio.

b) Blogs especializados en acompañar y analizar los avances del periodismo como profesión.

c) Páginas de este tipo que, sin estar adscritos a ningún medio digital, realizan una cobertura y análisis sistemático de los acontecimientos de actualidad, preferentemente de información general.

En nuestro trabajo, una vez superadas las primeras dudas identitarias del formato, hablamos de $j$-blogs del primer grupo establecido por Salaverría. Cumplen con todos los requisitos de la definición más extendida de blog ${ }^{1}$, aunque no son propiamente

1 Según Fundeu: "Puede usarse también bitácora o ciberdiario". Un blog, abreviatura del inglés weblog, según el Diccionario panhispánico de dudas es un "sitio electrónico personal, actualizado con mucha frecuencia, donde alguien escribe a modo de diario o sobre temas que despiertan su interés, y donde quedan recopilados asimismo los comentarios que esos textos suscitan en sus lectores. La traducción que se ha hecho de este término en español es bitácora, cuaderno de bitácora, ciberbitácora o ciberdiario pero el uso ya ha asentado en nuestra lengua el término blog escrito en redonda, es decir, sin comillas ni cursiva. Plural: blogs." 
páginas personales, pues el diario que las cobija y que paga al periodista está detrás. ¿Convierte esta circunstancia a estos blogs en columnas digitales?

Ramón Salaverría se lamenta en el trabajo citado de la carencia de referencias bibliográficas, algo que desconcierta en un campo tan fecundo como es el del estudio de los géneros (desde Martínez Albertos, 1983, y Martín Vivaldi, 1986, a Fernández Parratt, 2001; pasando por Gomis, 1989; Casasús y Núñez Ladevéze, 1991 entre otros). Ni siquiera el Manual de Redacción Ciberperiodística realiza un análisis específico, pese a estudiar pormenorizadamente la traslación de cada género al nuevo entorno digital. Describe las características de las bitácoras o weblogs, pero como un medio interactivo más (Díaz Noci y Salaverría, 2003: 295-299). Esa es otra cuestión, ¿son realmente interactivos los $j$-blogs?

\section{Objetivo, hipótesis y metodología}

Es un hecho convenido denominar blogs a las bitácoras profesionales asociadas a una cabecera periodística digital. Resulta pertinente insistir en la terminología, pues es una cuestión que tampoco ha estado exenta de debate. Bitácoras personales, grupales, profesionales, con múltiples posibilidades temáticas... Larrondo-Ureta (2005) recuerda que se han llegado a citar hasta veintiséis acepciones diferentes y por ello considera más ajustado para el investigador limitarse a ofrecer "algunas pinceladas útiles para recordar los rasgos estructurales básicos e identificativos de este tipo de publicación, caracterizada por su usabilidad a la hora de difundir de manera sencilla $-\mathrm{y}$, generalmente, gratuita- contenidos en red".

Una definición de consenso sería la siguiente: un blog es un sitio web personal o colectivo, con plataforma tecnológica preestablecida, que se actualiza periódicamente y recopila de forma cronológica inversa textos o artículos de una temática particular. Está abierto a los comentarios y el autor puede dirigir la conversación y darles respuesta de forma que se establezca el diálogo.

Así pues, partimos de que la rápida incorporación -y total aceptación por la Real Academia de la Lengua- del término "blog" a nuestro lenguaje lleva implícito el riesgo de uniformizar con un mismo concepto páginas con elementos diferenciales notables. Esta circunstancia obliga, si no se quieren perder importantes matices descriptivos, a esa continua taxonomización de las distintas categorías de bitácoras que se pueden encontrar en la red (Larrondo-Ureta, 2005) y, a la vez, a plantear dudas razonables sobre la identidad del género tan polivalente cuando pierde su carácter de publicación digital personal e independiente y pasa a vincularse a un medio de comunicación.

Además de aclarar estas incógnitas, este trabajo pretende como objetivo principal averiguar si el patrón estilístico y discursivo de estas bitácoras obedece a criterios análogos a los utilizados por los géneros periodísticos argumentativos, también llamados “de opinión" (Núñez Ladevéze, 1995: 97-110).

En nuestro entorno "columna" es también un término polisémico (Paniagua, 2000: 80). Generalmente se entiende como un artículo de autor publicado con regularidad en un espacio predeterminado del periódico. María Jesús Casals recuerda con esta metonimia (toma la parte-el espacio que ocupa en el medio impreso- por el todo) se designa un artículo de opinión que puede ser "razonador o lo contrario, falaz; orien- 
tador o enigmático; analítico o pasional; enjuiciativo o narrativo; y siempre valorativo, subjetivo, porque no puede ser de otro modo" (Casals, 2000: 32).

Este trabajo analiza, en el marco de referencia explicado, si los blogueros de las redacciones actúan más como "columnistas", que aportan contexto y argumentación a la información del diario, que como ciberperiodistas ${ }^{2}$. Para ello se parte de dos hipótesis:

1. Los $j$-blogs elaborados por experimentados periodistas tradicionales muestran que los grandes medios han dado pasos para la integración redaccional (papelinternet). Sin embargo, la comprensión absoluta del mundo digital de estos profesionales no es aún efectiva.

2. Estas bitácoras repiten recursos estilísticos más cercanos al papel que a los usos digitales. Esto explicaría su parecido estructural con las columnas de opinión del medio impreso.

El objetivo del trabajo no es tanto ser exhaustivo en el tratamiento como profundizar sobre los distintos conceptos y fenómenos involucrados en la génesis de lo que Antonio Fumero denomina metafóricamente "Blog Bang". Antes de realizar el trabajo, la pregunta era: ¿estos blogs periodísticos podrían incluirse en el concepto que este autor tiene sobre la blogosfera? (Fumero, 2005).

Para el estudio empírico, se acotó un corpus con todas las entradas realizadas durante el mes de mayo de 2012 en un amplio muestrario compuesto por 30 blogs alojados en tres diarios españoles de referencia, El País, El Mundo y La Vanguardia, y procedió a un análisis cualitativo/cuantitativo de los mismos. Quedaron fuera las bitácoras que, aun siendo de colaboradores habituales de estos medios, no están elaborados por periodistas tradicionales, como es el caso del Blog de Paul Krugman en Elpais.com, realizado por un economista que, además, no forma parte de la redacción. Este es un punto importante, porque para la selección de los j-blogs testigo se buscó un perfil de profesionales en activo, de gran experiencia y siempre vinculados laboralmente a cada redacción.

A partir de este doble condicionamiento de profesionalidad y vinculación efectiva a la redacción de papel se obtuvo el siguiente universo de estudio:

Tabla 1: Blogs testigo de Elmundo.es. Elaboración propia.

\begin{tabular}{|c|c|c|c|}
\hline Bitácora & Autor & Sección & Comienzo \\
\hline Blog de pecho & Rubén Amón & Blogs Cultura & $a b r-11$ \\
\hline ContraOpa & Carlos Segovia & Blogs Economia & sep-11 \\
\hline El selector & Custodio Pastor & Blogs & $a b r-12$ \\
\hline Entre Bits\&Chips & Javier López Tazón & Blogs Tecnología & feb-12 \\
\hline Espíritus animales & Pablo Pardo & Blogs Economía & ene-11 \\
\hline La hora de la verdad & Zabala de la Serna & Blogs Toros & feb-10 \\
\hline La sombra del poder & Lucia Méndez & Blogs & oct-10 \\
\hline Rock \& Blog & Quico Alsedo & Blogs & ene-10 \\
\hline
\end{tabular}

${ }^{2}$ La investigación se presentó en la mesa "Innovación. La web 2.0 y 3.0." del Congreso Complutense CUICIID 2012 bajo el título "Los nuevos 'j-blogs' asociados a diarios de prestigio se alejan de la concepción tradicional de bitácora. Treinta ejemplos en El País, El Mundo, y La Vanguardia". 


\begin{tabular}{|l|l|l|l|}
\hline $\begin{array}{l}\text { Sin noticias de Dior } \\
\text { Soñar despierto }\end{array}$ & $\begin{array}{l}\text { Beatriz Miranda } \\
\text { Alberto Luchini }\end{array}$ & Blogs Gentes! de verano & ene-10 \\
\hline \hline
\end{tabular}

Tabla 2: Blogs testigo de Elpaís.com. Elaboración propia.

\begin{tabular}{|c|c|c|c|}
\hline Crónica negra & Javier Valenzuela & Blogs Internacional & nov-11 \\
\hline Del alfiler al elefante & Lluis Basset & Blogs Internacional & abr-07 \\
\hline Despejen la sala & José Yoldi & Blogs Politica & jul-05 \\
\hline El ala Norte & Luis Rguez. Aizpeolea & Blogs Política & oct-11 \\
\hline El ojo izquierdo & Jose Ma Izquierdo & Blogs Política & feb-10 \\
\hline El Sur & Francisco Peregil & Blogs Internacional & $a b r-12$ \\
\hline Entre moquetas & Javier Casqueiro & Blogs Politica & oct-11 \\
\hline Información privilegiada & Miguel Ángel Noceda & Blogs Economia & ene-12 \\
\hline Puertas abiertas & Berna G. Harbour & Blogs Internacional & mar-12 \\
\hline Trigo limpio & Vidal Maté & Blogs Economía & ene-12 \\
\hline
\end{tabular}

Tabla 3: Blogs testigo, Lavanguardia.com. Elaboración propia.

\begin{tabular}{|c|c|c|c|}
\hline Cajón de sastre & Javier Dale & Blogs Actualidad & sep-11 \\
\hline Carta de Galicia & Anxo Lugilde & Blogs Actualidad & feb-09 \\
\hline Diario de Beirut & Tomás Alcoverro & Blogs Corresponsales & jul-02 \\
\hline Diario de Sao Paulo & Fernando Garcia & Blogs Corresponsales & jul-11 \\
\hline Diario de Valencia & Salvador Enguix & Blogs Actualidad & ene-06 \\
\hline El hilo & Xavier Mas de Xaxás & Blogs Actualidad & jul-09 \\
\hline El otro escaño & Iñaki Ellakuria & Blogs Actualidad & ene-11 \\
\hline Fem un reset & David González & Blogs Actualidad & jun-11 \\
\hline Pasos perdidos & Josep Gisbert & Blogs Actualidad & dic-10 \\
\hline Post Barcelona Shù & Jaume V. Aroca & Blogs Actualidad & jun-11 \\
\hline
\end{tabular}

El periodo de tiempo durante el que se realizó el trabajo de campo habría de resultar suficiente como para obtener análisis significativos y estandarizados, por lo que, una vez observado el ritmo de publicación y teniendo en cuenta que hablamos de blogs vinculados a un diario digital, se consideró que para obtener un volumen suficiente de ítems sobre los que llevar a cabo el análisis bastaba con un periodo de medio mes. Con el fin de reforzar la homogeneidad del estudio se prefirió que durante este tiempo no coincidieran acontecimientos informativos extraordinarios que pudiesen modificar el resultado. También se cuidó de que la época no coincidiera con periodos vacacionales, puesto que al ser blogs redaccionales podrían verse alterados. Con estos criterios, el periodo analizado fue el comprendido entre las 0:00 horas del primero de mayo de 2012 y las 0:00 horas del día 31 del mismo mes.

\section{Desarrollo de la investigación}

Una vez recabados los datos, se llevó a cabo un análisis cuantitativo de algunas de las variables de interpretación de los ítems con anotaciones extraídas durante el periodo de estudio.

El primer parámetro observado en la muestra es el de la frecuencia de publicación, que se muestra más propia de un columnista de medio impreso que de un blog personal, actualizado incluso varias veces en un mismo día. José Luis Orihuela insiste 
mucho en que, además de contenidos de calidad, fuentes relevantes, criterio en el uso de enlaces y respeto por los lectores, un blog requiere publicación frecuente. La media en nuestro caso es de poco más de una entrada por semana.

También se analiza en el promedio de palabras utilizadas para titular. Se trata de un elemento clave para detectar si el modelo utilizado es adecuado para la pantalla. Un título en la red ha de ser más descriptivo y mostrar desde el primer momento de qué se va a hablar. No existen los apoyos visuales del periódico. Salaverría señala que "la titulación periodística tradicional recurre esencialmente a dos modelos: uno relativamente extenso -en torno a 10 palabras- para los títulos informativos propios de noticias y otros géneros informativos, y otro más conciso -como máximo 6 ó 7 palabras, aunque frecuentemente menos- para los títulos apelativos o creativos, empleados habitualmente en géneros interpretativos y editorializantes", por lo que podemos inferir que en nuestro caso las entradas se alejan en extensión de lo habitual en los blogs. Se acercan, por el contrario, a lo que, trasladado al medio impreso estándar, al que de nuevo imitan, equivaldría más o menos a una columna de opinión. En un análisis pormenorizado de cada ficha se comprueba también que los títulos no son informativos ni se adaptan al estilo requerido en pantalla, pues el usuario -ni siquiera el habitual del blog- va a saber de qué trata la entrada hasta que no se haya sumergido en su lectura.

En los 30 blogs analizados, la extensión media de los títulos es de 5 palabras, coincidente con la titulación creativa de la que habla Salaverría. Los de mayor extensión corresponden a una forma más descriptiva. Además, en ningún caso se utiliza el característico estilo de titular en los periódicos que consiste en reproducir actos de habla (Núñez, 1995: 67-68). Al tratarse de profesionales en activo en el papel podría haberse deslizado el estilo que usan en la redacción habitualmente y que, como se sospecha (Pérez, 2012) entiendan el blog como una columna de opinión tradicional. Esta hipótesis se ve corroborada por la paupérrima utilización del hipertexto: nada menos que 14 de los 30 blogs no utiliza enlaces. Los 16 restantes muestran una utilización muy irregular, que más que del medio depende del propio periodista.

Si se tiene en cuenta que el hipertexto muestra "sin lugar a dudas" la destreza digital del bloguero y que el enlace es materia prima de las bitácoras pioneras (Rengel, 2005), los enlaces son un indicador de gran importancia, comparable según esta autora a la que pueda tener la habilidad lingüística. El trabajo arroja una media de dos enlaces por entrada, pero enseguida se puede descubrir que se trata de una generosa mentira estadística, pues hay casos como el de Custodio Pastor (El Selector) que elevan la media: introduce 41 enlaces en 5 post.

Otros elementos importantes para medir las habilidades ciberperiodísticas de los blogueros son el uso del hipertexto, la utilización de audio y vídeo y, por supuesto, la interacción con los usuarios por parte del periodista bloguero. De nuevo, el uso depende más del periodista que del medio.

\section{Conclusiones}

Las conclusiones permiten deducir que el patrón estilístico y discursivo en estas bitácoras es análogo al utilizado por los géneros periodísticos argumentativos tradicionales. Tal y como se sospechaba, se comportan como columnas digitales de opinión. 
Además, se trata de bitácoras que cuentan con suficientes singularidades e hibridaciones de género como para diferenciarlas radicalmente de la taxonomía habitual que se hace de los blogs. Sus contenidos son en todos los casos opinativos y obedecen a una retórica argumentativa en la que no se difunden datos novedosos, sino que trabajan con éstos -proporcionados por el propio diario o por otros medios, generalmente extranjeros- aplicando sus ideas, análisis y deducciones. Esta circunstancia queda reflejada tanto en la extensión de las entradas como en la longitud y forma de titular, poco descriptivos y cercanos al estilo utilizado en el diario para la opinión.

Los periodistas blogueros actúan como columnistas y, aunque han cambiado el papel del diario por una plataforma digital, apenas utilizan elementos multimediáticos (pocos vídeos y ningún audio). Además, el tono en todos los casos analizados tiende a editorializar en reseñas, glosas y comentarios tomados de otros medios o del propio diario. Tampoco responden a los comentarios de los usuarios, por lo que desaprovechan las oportunidades del medio digital.

Son estos quienes establecen conversación entre ellos, siguiendo el hilo de lo expuesto por el periodista, aunque en muchas ocasiones ajenos a lo que él dijo. La línea de opinión es la del medio por lo que, como sugiere Larrondo-Ureta, "no todo el mundo se muestra conforme a la hora de bautizar de blogging a esta práctica que inserta el blog en los medios de Internet" (Larrondo-Ureta, 2005). Si a todo ello se suma los demás elementos analizados, como la frecuencia de publicación, los títulos o la extensión de las entradas, la catalogación de los j-blogs se aleja de la blogosfera tradicional descrita por Antonio Fumero (2005). Está, sin duda, más cerca del periodismo tradicional disfrazado de digital.

\section{Referencias bibliográficas}

BLOOD, Rebecca (2002): The Weblog Handbook. Cambridge, MA. USA. Perseus Books Group.

CASALS CARRO, Ma Jesús (2000): "La columna periodística: de esos embusteros días del ego inmarchitable", en Estudios sobre el mensaje periodístico, $\mathrm{n}^{\circ}$ 6. Madrid, Servicio de Publicaciones de la Universidad Complutense.

CASASÚS, Josep Maria; Núñez Ladevéze, Luis (1991): Estilo y géneros periodísticos. Barcelona, Ariel.

CEBRIÁN, Mariano y FLORES, Jesús (eds., 2007): Blogs y periodismo en la Red. Madrid, Fragua.

DÍAZ NOCI, Javier y SALAVERRÍA, Ramón (coords., 2003): Manual de Redacción Ciberperiodística. Ariel. Barcelona.

EDO, Concha (2002): "El periodismo profesional y el desafío de los blogs". VII Congreso de la Sociedad Española de Periodística. Disponible en: http://bocc.ubi.pt/pag /edo-concha-periodismo-blogs.html\#tex $2 \mathrm{html} 1$ [Consultado: 26 de marzo de 2012]

EDO, Concha (2009): "La noticia en internet: cibermedios, blogs y entornos comunicativos emergentes" Estudios de Periodística XV. SEP. Madrid. Disponible en: http://www.bocc.ubi.pt/pag/edo-concha-internet-como-soporte-informativo.pdf [Consultado: 26 de marzo de 2012] 
ESTALELLA, Adolfo (2005): “Anatomía de los blogs". Telos, n 65, octubre-diciembre. Disponible en: http://sociedadinformacion.fundacion.telefonica.com/telos /articulocuaderno.asp@idarticulo\%3D9\&rev\%3D65.htm [Consultado el 1 de abril de 2012]

FERNÁNDEZ PARRAT, Sonia (2001): "El debate en torno a los géneros periodísticos en la prensa: nuevas propuestas de clasificación". Zer, no 11, pp. 293-310. Disponible en: http://www.ehu.es/zer/hemeroteca/pdfs/zer11-12-fernandez.pdf [Consultado: 20 de marzo de 2012]

FLORES, Jesús Miguel y AGUADO, Guadalupe (2006): “Claves de los weblogs y su influencia en la prensa tradicional". Doxa Comunicación, no 4, pp. 141-160. Disponible en: http://www.uspceu.com/usp/doxa/doxaIV/weblogs.pdf [Consultado: 20 de marzo de 2012]

FUMERO, Antonio (2005): "El abecé del universo blog", en Telos $n^{\circ} 65$, octubre-diciembre. Disponible en: http://sociedadinformacion.fundacion.telefonica.com/telos /articulocuaderno.asp@idarticulo\%3D1\&rev\%3D65.htm [Consultado: 15 de marzo de 2012]

GARCÍA, Berta y CAPÓN, José Luis (2004): "Las bitácoras o weblogs y la lógica del campo informativo. Un análisis comparativo de la agenda mediática tradicional". Estudios sobre el mensaje periodístico, $\mathrm{n}^{\mathrm{o}}$ 10: pp. 113-128. Madrid, Servicio de Publicaciones de la Universidad Complutense.

GOMIS, Lorenzo (1989): Teoría dels géneres periodistics. Barcelona, CIC.

GILLMOR, Dan (2004): We the Media. Grassroots Journalism by the People, for the People. O'Reilly Media Inc. Sebastopol, CA. EE.UU. Disponible en: http://oreilly.com/openbook/wemedia/book/index.html [Consultado: 22 de marzo de 2012]

LARRONDO-URETA, Ainara (2005): Presencia del formato weblog en los cibermedios: una aproximación a sus usos y funciones. Revista Latina de Comunicación Social, 60. La Laguna (Tenerife). Disponible en: http://www.ull.es/publicaciones/latina/200539larrondo.htma [Consultado: 19 de marzo de 2012]

LÓPEZ GARCÍA, Guillermo (2005): Modelos de comunicación en internet. Valencia, Tirant Lo Blanch.

MARCELO, Juan F.; MARTÍN, Eva (2007): La guía de bolsillo de los blogs. Madrid, Pearson.

MARTÍN VIVALDI, Gonzalo (1986): Géneros periodísticos. Madrid, Paraninfo.

MARTÍNEZ ALBERTOS, José Luis (1983): Curso general de redacción periodística. Barcelona, Paraninfo.

MORÁN, Próspero (2005): "Un acercamiento crítico a la consideración del weblog como género periodístico". Razón y palabra, $\mathrm{n}^{\circ} 48$. Disponible en: http://www.razonypalabra.org.mx/anteriores/n48/bienal/mesa14.pdf [Consultado el 20 de marzo de 2012] 
NIEMAN FOUNDATION (2003): "Nieman Reports" Nieman Foundation for Journalism at Harvard: Vol. 57, n⿳3, pp. 59-98 Disponible en: http://www.nieman.harvard.edu/reportsitem.aspx?id=101027 [Consultado el 15 de marzo de 2012]

NOGUERA VIVO, José Manuel (2008): Blogs y Medios. Las claves de una relación de interés mutuo. Montevideo, Libros en Red.

NÚÑEZ LADEVEZE, Luis (1995): Introducción al periodismo escrito. Barcelona, Ariel.

ORIHUELA, José Luis (2005): "Los weblogs y su identidad como cibermedios". En: SALAVERRÍA, Ramón (coord.): Cibermedios. El impacto de internet en los medios de comunicación en España. Sevilla, Comunicación Social, pp. 70-82.

PALOMO, Bella y MESO AYERDI, Koldo (2009): "Perfil y comportamiento de los autores de los blogs destacados en los ciberdiarios de Vocento". Anàlisi. Núm. 38, pág. 99-116. Disponible en: http://ddd.uab.es/pub/analisi/02112175n38p99.pdf [Consultado: 14 de marzo de 2012]

PÉREZ BAHÓN, Félix (2012): "Segunda oleada de blogs redaccionales en Elpais.com: utilización empresarial y asentamiento del formato como género argumentativo". XVIII Congreso Sociedad Española de Periodística. Universidad Carlos III de Madrid, 1 de junio. Getafe.

RENGEL, Irene (2005): "La utilización periodística y la integración del formato blog en diarios digitales europeos tradicionales. Los casos de 'elmundo.es' y 'Guardian Unlimited"'. Athenea Digital, no 8, otoño, pp. 348-369. Disponible en: http://redalyc.uaemex.mx/redalyc/pdf/537/53700812.pdf [Consultado: 22 de marzo de 2012].

SÁNCHEZ VIGIL, Juan-Miguel; RECIO, Juan Carlos; y OLIVERA-ZALDUA, María (2010): "Presencia y contenidos de los blogs en los principales diarios españoles". El Profesional de la Información, n6 noviembre-diciembre, pp. 602 609.

SALAVERRÍA, Ramón (coord., 2005): Cibermedios. El impacto de internet en los medios de comunicación en España. Sevilla, Comunicación Social, pp. 70-82.

SALAVERRÍA, Ramón (2008): "El estilo del blog periodístico: usos redaccionales en diez bitácoras españolas de información general". Actas y Memoria Final del Congreso Internacional Fundacional AE-IC (CD-Rom). Santiago de Compostela.

\section{Félix PÉREZ BAHÓN}

Universidad Carlos III de Madrid

Profesor Asociado de Periodismo

bahon30@gmail.com 